\title{
Trends in the use of computerized physician order entry by health-system affiliated ambulatory clinics in the United States, 2014-2016
}

Shira H. Fischer ${ }^{1 *}$ D, Robert S. Rudin ${ }^{1}$, Yunfeng Shi ${ }^{2}$, Paul Shekelle ${ }^{3,4}$, Alejandro Amill-Rosario ${ }^{2}$, Dennis Scanlon ${ }^{2}$ and Cheryl L. Damberg ${ }^{3}$

\begin{abstract}
Background: Computerized provider order entry (CPOE) can help providers deliver better quality care. We aimed to understand recent trends in use of CPOE by health system-affiliated ambulatory clinics.

Methods: We analyzed longitudinal data (2014-2016) for 19,109 ambulatory clinics that participated in all 3 years of the Healthcare Information and Management Systems Society Analytics survey to assess use of CPOE and identify characteristics of clinics associated with CPOE use.

We calculated descriptive statistics to examine overall trends in use, location of order entry (bedside vs. clinical station), and system-level use CPOE across all clinics. We used linear probability models to explore the association between clinic characteristics (practice size, practice type, and health system type) and two outcomes of interest: CPOE use at any point between 2014 and 2016, and CPOE use beginning in 2015 or 2016.

Results: Between 2014 and 2016, use of CPOE increased more than 9 percentage points from 58 to 67\%. Larger clinics and those affiliated with multi-hospital health systems were more likely to have reported use of CPOE. We found no difference in CPOE use by primary care versus specialty care clinics. When used, most clinics reported using CPOE for most or all of their orders. Health systems that used CPOE usually did so for all system-affiliated clinics.
\end{abstract}

Conclusions: Small practice size or not being part of a multi-hospital system are associated with lower use of CPOE between 2014 and 2016. Less than optimal use in these environments may be harming patient outcomes.

Keywords: CPOE, Computerized provider order entry, Ambulatory clinics, Health systems

\section{Background}

As hospitals and clinics have moved to adopt electronic health records (EHRs), the use of computerized provider order entry (CPOE) has grown considerably [1]. This upward trend in CPOE use aligns with federal policies designed to encourage the adoption of health information

\footnotetext{
* Correspondence: sfischer@rand.org

${ }^{1}$ RAND Corporation, 20 Park Plaza, Suite 920, Boston, MA 02116, USA

Full list of author information is available at the end of the article
}

technology (IT) to improve the quality of care; early studies in the hospital setting found that CPOE use was associated with improvements in safety, as well as with improved efficiency and reimbursement $[2,3]$. Specifically, the 2009 Health Information Technology for Economic and Clinical Health (HITECH) Act and Meaningful Use offered incentives to eligible providers, including those in ambulatory care settings, to adopt health IT. Since the HITECH Act, there has been a massive increase in EHR

(C) The Author(s). 2020 Open Access This article is licensed under a Creative Commons Attribution 4.0 International License, which permits use, sharing, adaptation, distribution and reproduction in any medium or format, as long as you give appropriate credit to the original author(s) and the source, provide a link to the Creative Commons licence, and indicate if changes were made. The images or other third party material in this article are included in the article's Creative Commons licence, unless indicated otherwise in a credit line to the material. If material is not included in the article's Creative Commons licence and your intended use is not permitted by statutory regulation or exceeds the permitted use, you will need to obtain permission directly from the copyright holder. To view a copy of this licence, visit http://creativecommons.org/licenses/by/4.0/ The Creative Commons Public Domain Dedication waiver (http://creativecommons.org/publicdomain/zero/1.0/) applies to the data made available in this article, unless otherwise stated in a credit line to the data. 
adoption in general and CPOE adoption in particular, especially in the hospital setting: the Agency for Healthcare Research and Quality reports that $84 \%$ of non-federal acute-care hospitals had implemented an EHR that included CPOE by the end of 2015, which is the most recent published data [4].

In contrast, data are sparse regarding ambulatory practice use of health IT. One form of CPOE is eprescribing, and at the end of 2015, studies of national samples suggested that slightly more than half of ambulatory practices had adopted EHRs with e-prescribing capabilities [5, 6]. However, little is known about CPOE use beyond medication prescribing as well as the factors associated with adoption in the ambulatory setting.

To understand the current state of CPOE use and recent trends in use by ambulatory practices, we used data from a national survey of health system-affiliated ambulatory clinics to examine current rates of use of $\mathrm{CPOE}$ and trends in adoption over a 3-year period and the association between CPOE use and clinic characteristics (practice size, practice type, and participation in a multihospital system).

\section{Methods}

CPOE includes computerized ordering of imaging tests, laboratory orders, referrals to other providers, and sometimes also electronic medication prescribing, though eprescribing is often studied as a separate functionality. In Meaningful Use criteria [7], as well as in the survey data we use (see below), e-prescribing is also measured by separate items. We focus in this paper on the Healthcare Information and Management Systems Society (HIMSS) Analytics Ambulatory Survey (HIMSS Analytics LOGIC ${ }^{\mathrm{mi}}$ Market Intelligence Platform) questions that ask about $\mathrm{CPOE}$ and not the specific medication ordering questions, and we interpret the survey questions about CPOE to include laboratory and other tests and referrals that a doctor can order.

\section{Data source}

We used three-year panel data (2014-2016) from the annual HIMSS Analytics Ambulatory Survey. This is a companion survey to the original HIMSS hospital survey, which has been used in studies of HIT and CPOE adoption [8-10]. The ambulatory survey includes clinics defined as facilities that provide "preventative, diagnostic, therapeutic, surgical, and/or rehabilitative outpatient care where the duration of treatment is less than 24 hours-and is generally referred to as outpatient care."

The survey captures information on more than $75 \%$ of U.S. health system-associated ambulatory care practices [11]. HIMSS defines a health system as an organization including at least one hospital and its associated nonacute facilities, where "associated" indicates a governance relationship (i.e., owned, leased, or managed by a health system). The survey includes approximately 42,000 clinics across the United States.

\section{Approach}

We limited our datasets to health system-associated ambulatory clinics that deliver primary or specialty care, eliminating imaging centers or other locations where ordering was not expected. We removed observations that were missing entries for predictor variables. When adoption patterns seemed illogical or inaccurate-for example, if a clinic was noted as using CPOE in 2014 and 2016 but not in 2015, or if an answer did not match the number of answer choices, we considered the responses as probably incorrect and dropped the clinic from our sample. This logic-checking resulted in eliminating 2169 records, or about $10 \%$, with a final sample size of 19,109 clinics.

\section{Clinic characteristics}

Using HIMSS survey data, we classified ambulatory clinics according to size (based on number of physicians, dichotomized into 3 or fewer vs. $>3$ ); clinic type (primary versus specialty), and health system type (single hospital or multi-hospital health system).

\section{Study outcome}

We measured two outcomes, CPOE use and the frequency of that use, that were collected in the HIMSS survey. The survey first asks clinics about how EHR software is being used, with a checkbox option for "Clinician Order Entry," among other options. Within the Clinician Order Entry question response options, respondents could indicate location where CPOE was used (i.e., whether the functionality was available at a clinician station or at the point of care) with both options potentially being selected. We created a single variable that represented overall use, whether the overall question or any location was selected, and we used this variable for most of our analyses.

The second relevant question focused on percent of CPOE use, titled "CPOE - \% of Medical Orders Entered by Physicians." If this option was checked off in the main column, the respondent was asked to estimate a percentage of medical orders that were entered electronically by physicians. There were five options: $1-25 \%$ of orders, $26-50 \%$ of orders, $51-75 \%$ of orders, $76-94 \%$ of orders, or $>94 \%$ of orders. We used these findings for analyses about percentage of orders.

The survey did not clarify whether CPOE was to include e-prescribing, and there were other medication management questions on the survey, so we did an 
additional analysis on the questions on electronic medication prescribing (namely, two checkboxes for "E-Prescribing new medications" and "E- Prescribing refill medication requests") as a comparison, described below.

\section{Analysis}

We computed descriptive statistics, reporting the mean CPOE use rate by year (2014-2016) and by clinic type.

We used a multivariable linear probability model to examine the association between use of CPOE by 2016 and three clinic characteristics available in the HIMSS database: size (based on number of physicians, dichotomized into 3 or fewer vs. > 3); clinic type (primary versus specialty), and whether the clinic was part of a health system, and if so what system type (i.e., single hospital or multi-hospital health system).

As a sensitivity analysis, we examined the factors associated with changes in adoption status. With the subsample of clinics that did not have CPOE in 2014, we used multivariable linear probability models to analyze the relationship between size, clinic type, and health system type and new adoption (i.e., clinics newly adopting CPOE in 2015 or 2016). In all regression analyses, we clustered standard errors at the health system level to account for multiple clinics associated with the same health system.

We then examined use of CPOE by clinics within health systems by calculating the number of health systems with full adoption (all affiliated clinics used CPOE), partial adoption (some clinics used CPOE while others did not), or no adoption (no clinics within a system used CPOE).

As additional analyses, we explored factors predicting use of electronic prescribing of medications, to see if there were different predictors for this related functionality, which is a form of CPOE restricted to medications, and we also compared data exchange over time and among those with CPOE and without.

\section{Results}

In 2016, our study sample had 19,109 clinics within 1548 systems (Table 1). The majority of ambulatory clinics had EHRs (88\% by $2014 ; 96 \%$ in 2016). The number of clinics per health system also increased over this time period. The total number of health systems decreased by 109 (almost 7\%), with the number of clinics per system increasing, likely reflecting consolidation over the study period. The portion of primary care clinics was stable at around $65 \%$. An increasing proportion of clinics were part of a multihospital system, again possibly reflecting mergers and acquisitions. Characteristics of ambulatory clinics and the health systems in which they exist are shown in Table 1.

\section{CPOE use}

Overall CPOE use increased between 2014 and 2016, from 58 to $67 \%$. In the vast majority of ambulatory clinics, CPOE was available both at the bedside and at the clinician station ( $88 \%$ in 2014 to $92 \%$ in 2016). As Table 2 illustrates, clinics that were part of a multi-hospital system (vs. those affiliated with a single hospital) had $14 \%(p<0.0001)$ higher rates of CPOE use, while larger practices had an 8.7\% $(\mathrm{p}<0.0001)$ greater use of $\mathrm{CPOE}$ than smaller practices as defined by less than three physicians at the ambulatory site (see Table 2).

Slightly more than 8000 clinics had still not yet adopted CPOE in 2014. For this sub-sample, we examined the likelihood of adoption by 2016 and found the factors that predicted adoption in 2015-2016 were being part of a multi-hospital system (9.6\% increased likelihood, $p=0.024$ ) and being part of a larger practice $(8.9 \%$ increased likelihood, $p<0.0001)$.

Table 1 Characteristics of Ambulatory Clinics Included in the CPOE Study Sample (2014-2016)

\begin{tabular}{llll}
\hline Year & 2014 & 2015 & 2016 \\
\hline Number of clinics & 19,109 & 19,109 & 1603 \\
Number of health systems & 1657 & 1603 & 1109 \\
Number of clinics per health system (standard deviation) & $11.53(26)$ & $11.92(27)$ & $12.34(29)$ \\
Number of clinics that are Primary Care (\% of total clinics) & $12,439(65 \%)$ & $12,336(65 \%)$ & $12,296(64 \%)$ \\
Number of clinics in multi-hospital systems (\% of total clinics) & $13,301(69 \%)$ & $13,718(72 \%)$ & $13,887(72 \%)$ \\
Number of physicians per clinic (standard deviation) & $7.04(32)$ & $7.02(32)$ & $7.07(32)$ \\
Number of clinics with EHRs (\% of total clinics) & $16,910(88 \%)$ & $17,944(94 \%)$ & $18,386(96 \%)$ \\
Number of clinics with CPOE (\% of total clinics) & $11,049(58 \%)$ & $12,022(63 \%)$ & $12,785(67 \%)$ \\
Number of clinics in Primary Care Practices with EHRs (\% of Primary Care clinics) & $10,991(88 \%)$ & $11,551(94 \%)$ & $11,796(96 \%)$ \\
Number of clinics in multi-hospital systems with EHRs (as \% of multi-hospital systems clinics) & $12,020(90 \%)$ & $13,055(95 \%)$ & $13,500(97 \%)$ \\
\hline
\end{tabular}


Table 2 Characteristics Associated with CPOE Use (Through 2016) Across All Clinics in 3 Years (54,826 observations) - Multivariable Linear Regression Model

\begin{tabular}{|c|c|c|c|c|c|c|c|c|c|c|}
\hline \multirow[t]{2}{*}{ Variable: } & \multicolumn{2}{|l|}{ Primary care } & \multicolumn{2}{|l|}{ Multi-hospital system } & \multicolumn{2}{|c|}{ Size of practice (> 3 physicians vs. $0-3$ ) } & \multicolumn{4}{|l|}{ Year } \\
\hline & Estimated coefficient & $p$ value & Estimated coefficient & $p$ value & Estimated coefficient & $p$ value & 2015 & $p$ value & 2016 & $p$ value \\
\hline CPOE use & 0.028 & 0.055 & 0.14 & $<.0001$ & 0.087 & $<.0001$ & 0.050 & $<.0001$ & 0.089 & $<.0001$ \\
\hline
\end{tabular}

When looking at only those clinics with an EHR in $2014(88 \%)$, the predictive factors were the same (data not shown). Primary care clinic type was not significantly associated with CPOE adoption (see Table 3). At the health system level, the percent of systems with all clinics using $C P O E$ increased over time from 31 to $39 \%$, and fewer health systems (from 53 to 48\%) had no adoption among their clinics, with the some category remaining similar over the 3 years (see Fig. 1).

\section{Percent of orders placed via CPOE}

Among clinics that were using $\mathrm{CPOE}$, the majority (almost 60\%) indicated that CPOE was used for almost all orders. The proportion of clinics using CPOE for more than $94 \%$ of their orders increased from $26 \%$ in 2014 to $32 \%$ in 2016 , with a similar increase of about 6 percentage points in those using it for $75-94 \%$ of orders (see Table 4). Most of the change came from increased adoption (from clinics who were not using any CPOE at all) rather than an increase from clinics who had been using CPOE for only a small percent of orders. Very few clinics were using $\mathrm{CPOE}$ for less than $75 \%$ of their orders in
2014, with $8.9 \%$ doing so, a number that decreased to $7.3 \%$ in 2016. Among clinics with CPOE, in more than $90 \%$ of cases CPOE was taking place both at the point of care and at a clinician station.

\section{Electronic prescribing analysis}

We conducted a similar analysis for medication prescribing. The two measures of interest, electronic prescribing (measured separately for both new prescriptions and refill requests) and physician order entry, were highly correlated. Using 2016 data, the variables had a Pearson correlation of 0.90 for new prescriptions and 0.88 for refills, and at least $95 \%$ of the time, a clinic had either both (indicating CPOE and e-prescribing both being used) or neither.

Analysis of e-prescribing by clinic yielded similar results. E-prescribing for new medications and eprescribing for refill medication requests both increased over the 2-year period-from 56 to $64 \%$ for new medications and from 55 to $64 \%$ for refills. Regression analysis using linear probability demonstrated a significant relationship of e-prescribing to the kind of hospital system (those in a multi-hospital system were $16 \%$ more likely to use e-prescribing, $p<0.001$ ) and larger clinic size $(10 \%$ more likely, $\mathrm{p}<0.001)$. As

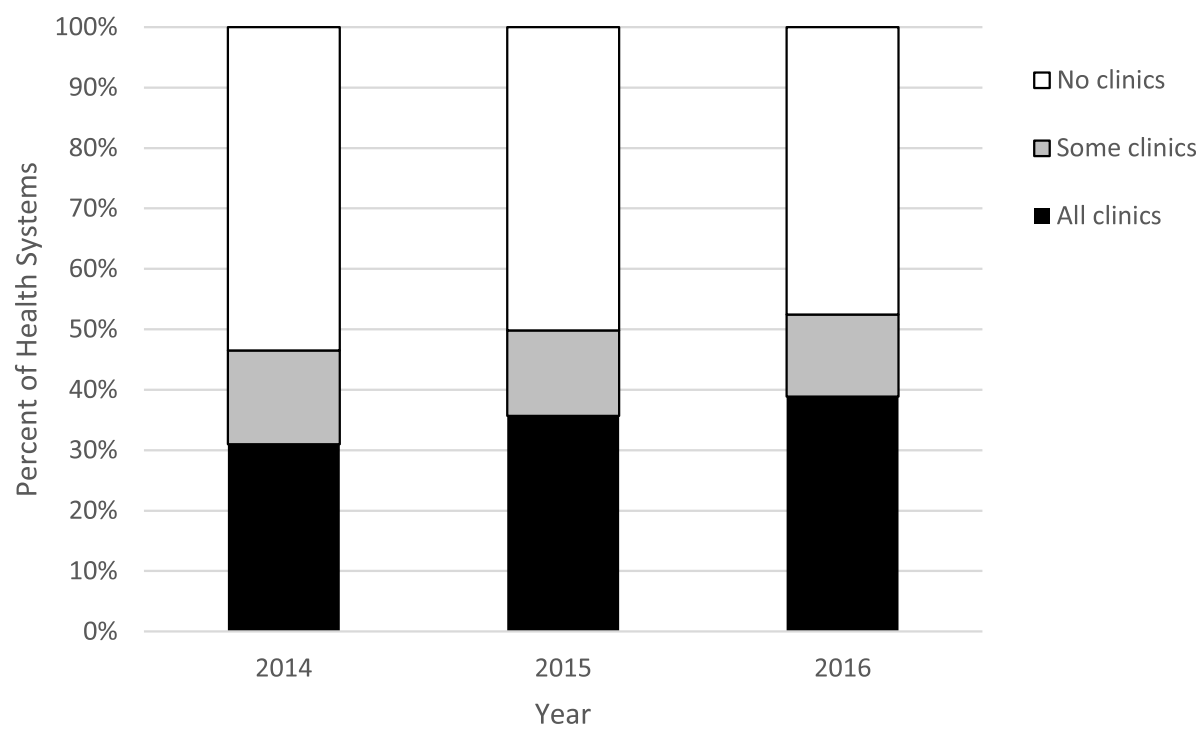

Fig. 1 Adoption of CPOE Within Systems, 2014-2016 (4808 systems over the 3 years) 
Table 3 Characteristics Associated with CPOE Adoption During Study Period (Nonusers in 2014 Who Adopted in 2015 or 2016) Compared to Non-Adopters (7843 observations) - Multivariable Linear Regression Model

\begin{tabular}{|c|c|c|c|c|c|c|}
\hline \multirow[t]{2}{*}{ Variable: } & \multicolumn{2}{|l|}{ Primary care } & \multicolumn{2}{|l|}{ Multi-hospital system } & \multicolumn{2}{|c|}{ Size of practice (> 3 physicians vs. $0-3$ ) } \\
\hline & Estimated coefficient & $p$ value & Estimated coefficient & $p$ value & Estimated coefficient & $p$ value \\
\hline CPOE adoption & 0.013 & 0.424 & 0.096 & 0.024 & 0.089 & $<.0001$ \\
\hline
\end{tabular}

with CPOE, primary care status was not a significant predictor of use. Full results of this analysis can be found in the Additional file 1.

\section{Information exchange}

We also found that CPOE is associated with health information exchange (HIE). Ability to exchange data across multiple vendor platforms for health information exchange (HIE) increased over the 3 years, from $31 \%$ in 2014 to $42 \%$ in 2016. Data exchange with government and hospitals also increased, from 36 to $48 \%$ and 46 to $56 \%$, respectively-meaning that almost half of clinics still do not exchange information with hospitals for clinical information, and as of 2016, only half (51\%) exchange data with other clinics for clinical information. When accounting for CPOE status, it is clear that those with electronic prescribing are also much more likely to exchange data in various ways; among the subset of our dataset that did not adopt $\mathrm{CPOE}$, the rate of exchange with hospitals, for example, was 3\%, compared to $80 \%$ among those with CPOE.

\section{Discussion}

Use of CPOE among health system-affiliated clinics increased between 2014 and 2016, though near-universal adoption of CPOE, which is now the norm in the hospital setting, is far from being achieved. Although virtually all ambulatory clinics (96\% by 2016) report having an EHR, only $65 \%$ reported having CPOE. Larger clinics and those affiliated with multi-hospital systems are ahead in adopting CPOE. Once adopted, CPOE quickly becomes the dominant form of ordering: clinics who have adopted it use it for more than $94 \%$ of their orders. Some health IT functionalities rely on CPOE, such as clinical decision support (CDS), so it makes sense that it would often be adopted first. Exchange of data is also associated with CPOE adoption. The benefit of CPOE on

Table 4 Percent of Orders Placed Using CPOE, 2014-2016

\begin{tabular}{llll}
\hline & 2014 & 2015 & 2016 \\
\hline None & $10,564(55.28 \%)$ & $9549(49.97 \%)$ & $8584(44.92 \%)$ \\
$1-25 \%$ & $113(0.59 \%)$ & $111(0.58 \%)$ & $122(0.64 \%)$ \\
$26-50 \%$ & $304(1.59 \%)$ & $271(1.42 \%)$ & $259(1.36 \%)$ \\
$51-75 \%$ & $1279(6.69 \%)$ & $1301(6.81 \%)$ & $1028(5.38 \%)$ \\
$75-94 \%$ & $1856(9.71 \%)$ & $2214(11.59 \%)$ & $2982(15.61 \%)$ \\
$>94 \%$ & $4993(26.13 \%)$ & $5663(29.64 \%)$ & $6134(32.10 \%)$ \\
\hline
\end{tabular}

patient outcomes is likely not to be realized without other functionalities.

Despite the near-universal (96\%) adoption of an EHR, about one-third of ambulatory clinics have not yet implemented one of the most basic EHR functionalities, CPOE. Adoption rates are lower in smaller practices and single-hospital systems. Therefore, policies promoting CPOE adoption should specifically target these practices. Without a bigger push, at the rate seen in these data, it will take at least another 6 years or more for CPOE to achieve the kind of use in ambulatory clinics that it already has achieved in hospitals. In particular, the "allor-none" finding of CPOE use by clinics within system suggests that an effective action might be to encourage systems to adopt CPOE. Furthermore, the $15 \%$ or so of practices that indicated only some clinics use CPOE suggests a heterogeneity in adoption; identifying the reason for this internal variation is tolerated could help determine how to increase uptake.

Our study has limitations. The dataset includes only health system-affiliated ambulatory clinics, and as such does not allow us to estimate the prevalence of use among non-system-affiliated clinics, which likely have lower rates of adoption. Even among health systemaffiliated ambulatory clinics the response rate to the survey was only $75 \%$, meaning inference from these results to the entire sample must be made with some caution. The HIMSS data are self-reported and not validated, and there may be favorable response bias (HIMSS surveys have been shown to have higher rates of adoption in the past) [12] though they represent $75 \%$ of relevant clinics, and at the level of major functionalities, one recent validity assessment showed reasonable accuracy [13]. The HIMSS data are also restricted to ambulatory clinics affiliated with a health system; the EHR data about unaffiliated clinics has only recently been released and is still incomplete. However, other surveys do not provide the level of detail that the HIMSS survey provides [14]. Another limitation is that the response options to the survey questions about CPOE were binary-use or notwith one question on estimates of use rates (percentage of orders). The survey did not include nuances of how CPOE was implemented, what kind of tools were available, or how it was used-for example, whether it was optional or required. When the HIMSS data on unaffiliated clinics are sufficiently mature, analyses across all ambulatory clinics will be possible. 


\section{Conclusion}

CPOE use is increasing in system-affiliated ambulatory practices, but adoption is still lower than desired, given federal efforts to promote the use of EHRs and the adoption of a broad set of health IT functionalities including CPOE. When adopted, clinics tended to use it for most or all of their orders. Systems tended to have homogenous adoption or non-adoption of CPOE by the clinics within their system. As policymakers work to advance the use of health IT, a critical gap area that requires attention are smaller clinics and those affiliated with single hospital system. To determine how to support expansion efforts, it will be important to learn why these clinics are not yet using CPOE.

\section{Supplementary information}

Supplementary information accompanies this paper at https://doi.org/10. 1186/s12913-020-05679-4.

Additional file 1. Additional analysis examining two medication-related outcomes: e-prescribing for new medications and e-prescribing for refill requests. $N=19,804$ clinics.

\section{Abbreviations}

CDS: Clinical decision support; CPOE: Computerized provider order entry; EHRs: Electronic health records; HIE: Health information exchange; IT: Health information technology; HITECH: Health Information Technology for Economic and Clinical Health; HIMSS: Healthcare Information and Management Systems Society

\section{Acknowledgements}

Not applicable

\section{Authors' contributions}

SHF, RSR, YS, PS, AA-R, DS, and CLD all contributed to the conception and design of the work. SHF and RSR designed and conducted the analyses with programming support. SHF drafted the manuscript. YS and PS aided with the approach and analysis. All authors read and approved the final manuscript.

\section{Funding}

This work was supported through the RAND Center of Excellence on Health System Performance, which is funded through a cooperative agreement (1U19HS024067-01) between the RAND Corporation and the Agency for Healthcare Research and Quality. The content and opinions expressed in this publication are solely the responsibility of the authors and do not reflect the official position of the Agency or the U.S. Department of Health and Human Services

\section{Availability of data and materials}

No patient data was used in this work. The data that support the findings of this study are available from HIMSS but restrictions apply to the availability of these data, which were used under license for the current study, and so are not publicly available. Data are however available from the authors upon reasonable request and with permission of HIMSS.

\section{Ethics approval and consent to participate}

Not applicable as this is secondary data.

\section{Consent for publication}

Not applicable

Competing interests

The authors declare that they have no competing interests.

\section{Author details}

${ }^{1}$ RAND Corporation, 20 Park Plaza, Suite 920, Boston, MA 02116, USA

${ }^{2}$ Pennsylvania State University, University Park, State College, PA, USA. ${ }^{3}$ RAND Corporation, 1776 Main St, Santa Monica, CA 90401, USA. ${ }^{4}$ West Los Angeles VA Medical Center, 11301 Wilshire Boulevard, Los Angeles, CA 90073, USA.

Received: 1 November 2019 Accepted: 21 August 2020

Published online: 07 September 2020

\section{References}

1. Pedersen CA, Schneider PJ, Scheckelhoff DJ. ASHP national survey of pharmacy practice in hospital settings: Prescribing and transcribing-2016. Am J Health Syst Pharm. 2017;74(17):1336-52.

2. Nuckols TK, Smith-Spangler C, Morton SC, et al. The effectiveness of computerized order entry at reducing preventable adverse drug events and medication errors in hospital settings: a systematic review and metaanalysis. Syst Rev. 2014;3:56.

3. Bates DW, Leape LL, Cullen DJ, et al. Effect of computerized physician order entry and a team intervention on prevention of serious medication errors. JAMA. 1998:280(15):1311-6.

4. The Office of the National Coordinator for Health Information Technology Health IT Dashboard: Non-federal Acute Care Hospital Electronic Health Record Adoption, 2015. 2018; Available at: https://dashboard.healthit.gov/ quickstats/pages/FIG-Hospital-EHR-Adoption.php. Accessed August, 2018.

5. Agency for Healthcare Research and Quality. Computerized Provider Order Entry. 2018; Available at: https://psnet.ahrq.gov/primers/primer/6/ computerized-provider-order-entry. Accessed August, 2018.

6. The Office of the National Coordinator for Health Information Technology. Office-based Physician Electronic Health Record Adoption, 2015. 2018; Available at: https://dashboard.healthit.gov/quickstats/pages/physician-ehradoption-trends.php. Accessed August, 2018.

7. Centers for Medicare \& Medicaid Services (CMS). Electronic Prescribing (eRx) Incentive Program. 2018; Available at: https://www.cms.gov/Medicare/ Quality-Initiatives-Patient-Assessment-Instruments/ERxIncentive/index.html. Accessed Nov 26, 2018.

8. Furukawa MF, Raghu TS, Spaulding TJ, Vinze A. Adoption of health information technology for medication safety in U.S. hospitals, 2006. Health Aff (Millwood). 2008;27(3):865-75.

9. McCullough JS. The adoption of hospital information systems. Health Econ. 2008;17(5):649-64.

10. Kharrazi H, Gonzalez CP, Lowe KB, Huerta TR, Ford EW. Forecasting the Maturation of Electronic Health Record Functions Among US Hospitals: Retrospective Analysis and Predictive Model. J Med Internet Res. 2018;20(8): e10458.

11. Rumball-Smith J, Shekelle P, Damberg CL. Electronic health record "superusers" and "under-users" in ambulatory care practices. Am J Manag Care. Jan 2018;24(1):26-31.

12. Burt CW, Hing E. Use of computerized clinical support systems in medical settings: United States, 2001-03. Adv Data. 2005;353:1-8.

13. Rudin RS, Shi Y, Fischer SH, et al. Level of agreement on health IT adoption and use in survey data: a mixed-methods analysis of ambulatory clinics in one U.S. state. JAMIA Open. In Press.

14. National Center for Health Statistics. Ambulatory Health Care Data: Survey Results and Products. 2017; Available at: https://www.cdc.gov/nchs/ahcd/ ahcd_products.htm. Accessed June 28, 2020.

\section{Publisher's Note}

Springer Nature remains neutral with regard to jurisdictional claims in published maps and institutional affiliations. 